\title{
Galactic spiral structure revealed by Gaia EDR3 ${ }^{\star}$
}

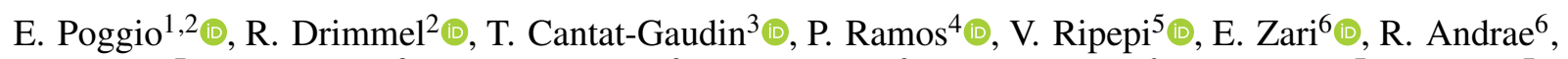

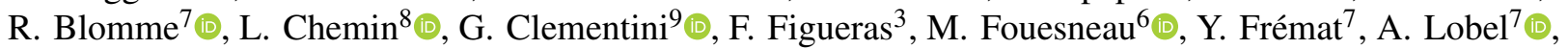 \\ D. J. Marshall ${ }^{10,11}$, T. Muraveva ${ }^{9}$, and M. Romero-Gómez ${ }^{3} \odot$
}

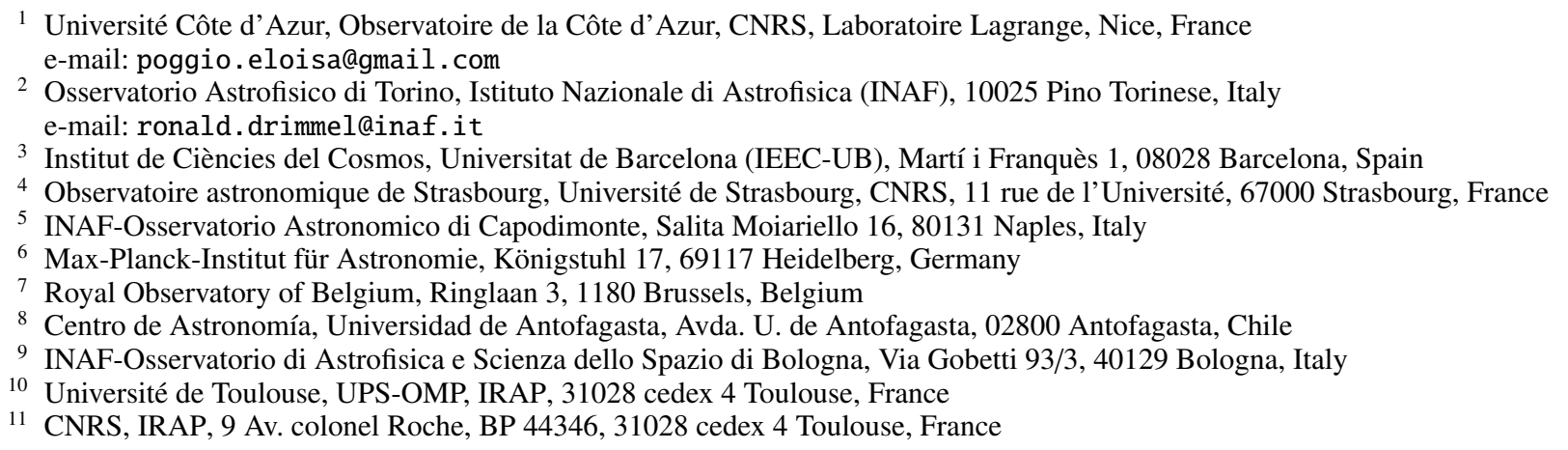

Received 1 March 2021 / Accepted 7 May 2021

\begin{abstract}
Using the astrometry and integrated photometry from the Gaia Early Data Release 3, we map the density variations in the distribution of young upper main sequence (UMS) stars, open clusters, and classical Cepheids in the Galactic disc within several kiloparsecs of the Sun. We derive maps of relative over- and under-dense regions for UMS stars in the Galactic disc using both bivariate kernel density estimators and wavelet transformations. The resulting overdensity maps exhibit large-scale arches that extend in a clumpy but coherent way over the entire sampled volume, indicating the location of the spiral arm segments in the vicinity of the Sun. Peaks in the UMS overdensity are well matched by the distribution of young and intrinsically bright open clusters. By applying a wavelet transformation to a sample of classical Cepheids, we find that their overdensities possibly extend the spiral arm segments on a larger scale ( $\simeq 10 \mathrm{kpc}$ from the Sun). While the resulting map based on the UMS sample is generally consistent with previous models of the Sagittarius-Carina spiral arm, the geometry of the arms in the III quadrant (galactic longitudes $180^{\circ}<l<270^{\circ}$ ) differs significantly from that suggested by many previous models. In particular, we find that our maps favour a larger pitch angle for the Perseus arm, and that the Local Arm extends into the III quadrant at least $4 \mathrm{kpc}$ past the position of the Sun, giving it a total length of at least $8 \mathrm{kpc}$.
\end{abstract}

Key words. Galaxy: disk - Galaxy: structure - Galaxy: stellar content

\section{Introduction}

The first indication of the large-scale structure of the Milky Way came with the realisation that it was but one of a large class of galaxies, of which our neighbour Andromeda served as the first archetype. At that point, our collective mental picture of the Milky Way was that of a spiral galaxy, though we remained ignorant of the number and position of the spiral arms. The first hint of spiral structure in the vicinity of the Sun was found in the distribution of short-lived high-mass stars and early $21 \mathrm{~cm}$ surveys (Morgan et al. 1952, 1953; Christiansen \& Hindman 1952) showing three spiral arm segments. The transparency of the interstellar medium to radio wavelengths soon provided confirmation that the distribution of gas in the disc had spiral arms on large scales (van de Hulst et al. 1954; Kerr et al. 1957; Oort et al. 1958), though we still lacked a clear picture.

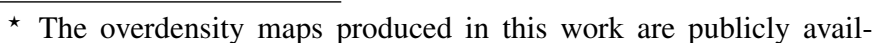
able at https://github.com/epoggio/Spiral_arms_EDR3.git. The list of cluster parameters is only available at the CDS via anonymous ftp to cdsarc.u-strasbg.fr $(130.79 .128 .5)$ or via http: //cdsarc.u-strasbg.fr/viz-bin/cat/J/A+A/651/A104
The large-scale spiral structure would become evident after considerable effort, in combining both radio and optical data of HII regions, with the definitive work of Georgelin \& Georgelin (1976). These authors convincingly traced out four spiral arms, and their mapping would later be improved and extended by Taylor \& Cordes (1993), Bland-Hawthorn et al. (2002), and Russeil (2003). Advances in $21 \mathrm{~cm}$ surveys and modelling also showed four arms in the outer galaxy, where kinematic distances are unambiguous, out to about $25 \mathrm{kpc}$ from the Galactic centre (Levine et al. 2006). More recently, Reid et al. (2014, 2019) mapped the spiral arms using absolute radio astrometry of maser sources, which are thought to be powered by young stellar objects. The spiral structure traced by the masers is again broadly consistent with the picture painted by the HII mapping of a four-armed spiral. Classical Cepheids also began to be used to trace the spiral arms on larger scales (Skowron et al. 2019; Veselova \& Nikiforov 2020). While these and most other models agree on the number of major arms in the Milky Way, as traced by star formation products, there are some differences in their position, shape, and pitch angle. The most marked difference is between the spiral arms in the outer disc of Levine et al. (2006), with a pitch angle of approximately $24^{\circ}$, and other 
models based on tracers in the inner Galaxy, which typically have pitch angles of between $12^{\circ}$ and $13^{\circ}$ (Vallée 2005). In particular, these models differ in their proposed tracing of the Perseus arm.

In most spiral arm models, the Local Arm, one of the three spiral arm segments detected and passing in close vicinity to the Sun, is either not present or is added as a small local feature, a relatively minor spur or arm segment. Nevertheless, due to its proximity, this feature dominates the observed distribution on the sky of bright young stars (Poggio et al. 2017). Only recently, with the mapping of radio masers associated with young stellar objects, has it become evident that the Local Arm extends further into the first quadrant (galactic longitudes $0^{\circ}>l>90^{\circ}$ ) than previously thought (Xu et al. 2016). Miyachi et al. (2019) found that the stellar Local Arm has a larger pitch angle compared to the one based on high-mass star forming regions from $\mathrm{Xu}$ et al. (2016). For recent reviews of our current knowledge of the spiral structure of the Milky Way, see Xu et al. (2018a) and Shen \& Zheng (2020).

With the advent of the Gaia mission (Gaia Collaboration 2016, 2018a, 2021) and its more-than 1 billion stellar parallaxes, there has been a resurgence in mapping the spiral structure in the vicinity of the Sun, as seen in young populations. CantatGaudin et al. (2018, 2020) and Kounkel et al. (2020) identified and mapped open clusters, while Xu et al. (2018b), Chen et al. (2019), and Xu et al. (2021) investigated the distribution of OB stars as well as relevant maser sources with radio astrometry. Combining Gaia astrometry with radial-velocity data, evidence of spiral arm structure is also found in the kinematics of stars in the form of non-circular streaming motions (Gaia Collaboration 2018b; Eilers et al. 2020; Khoperskov et al. 2020).

In this contribution we investigate the spiral arm structure, as seen in the surface density of three types of young stellar tracers: upper main sequence stars, open clusters, and classical Cepheids. The paper is structured as follows: in Sect. 2, we describe the data sets used in this work; the methods and results are presented in Sect. 3; we discuss our results in Sect. 4, and present our conclusions in Sect. 5.

\section{Data}

In the following sections, we give an overview of the data sets used in the present work.

\subsection{Upper main sequence stars}

Here we use two samples of upper main sequence (UMS) stars:

- The 'P18' UMS sample, which contains the UMS stars from Poggio et al. (2018, hereafter P18), but with Gaia EDR3 astrometry (Gaia Collaboration 2021). In P18, the selection of the stars was performed in two stages. First, a preliminary selection was made using photometric measurements from the 2Micron All Sky Survey (2MASS, Skrutskie et al. 2006) and Gaia (Evans et al. 2018), selecting stars with $G<15.5$ and colours consistent with intrinsically blue sources subject to reddening. The selection was further refined via a probabilistic approach based on Gaia astrometry, with the aim of selecting stars that are likely to be brighter than a given (reddened) absolute magnitude $M_{\text {lim }}$, chosen to be roughly consistent with a B3V-type star. For this sample, distances are recalculated using the Bayesian procedure described in P18, but using Gaia EDR3 astrometry corrected for the mean zero point parallax offset following Lindegren et al. (2021), resulting in 603787 selected sources.
Because of quality cuts on the 2MASS photometry, it was found that many nearby (bright) blue sources were missing. We therefore selected Gaia EDR3 sources with apparent magnitude $G<15.5$, colour $\left(G_{\mathrm{BP}}-G_{\mathrm{RP}}\right)<0$, and parallax $\varpi>$ 0.2 mas. Additionally, we only select stars satisfying the relation $G+5 \log (\varpi / 1000)+5<0$, to remove white dwarf contaminants. This procedure gave us 6827 stars, of which 3259 are not in the P18 sample described above. Of these, only 50 were found to have a parallax signal-to-noise ratio $(\mathrm{S} / \mathrm{N})$ smaller than 5 , and we excluded them from our sample. For the remaining 3209 stars, we simply invert the parallax to obtain their distance. In any case, as discussed below, the role played by these 3209 nearby UMS stars is almost irrelevant to the results presented in this contribution, as they only cause modest changes within $0.3-$ $0.4 \mathrm{kpc}$ of the Sun, while the aim of this work is to map the spiral structure of the Galaxy on a larger scale.

The final UMS P18 sample contains 606219 stars, after removing duplicated sources resulting from the cross-match between Gaia DR2 and DR3.

- The 'Z21' UMS sample, which contains the stars in the 'filtered' sample from Zari et al. (2021). Similarly to P18, Z21 selected their UMS initial sample by applying simple colour cuts combining Gaia DR3 and 2MASS photometry. To select intrinsically luminous stars (of spectral type earlier than $\sim \mathrm{B} 7 \mathrm{~V}$ ), they restricted their sample to stars with absolute magnitude in the 2MASS $K_{\mathrm{s}}$ band of $M_{K_{\mathrm{s}}}<0$ mag. Z21 refer to this sample as the 'target sample', as it is devised for spectroscopic follow up. Finally, Z21 cleaned their target sample of sources with spurious astrometric solutions using the classifier presented in Rybizki et al. (2021), and of sources that do not follow disc kinematics of a young stellar population. Their 'filtered' sample consists of 435273 stars. Z21 estimated distances using a model designed to reproduce the properties of their data set in terms of its spatial and luminosity distribution, and the additional criterion that stars belonging to their sample should follow Galactic rotation, with a small, typical velocity dispersion. These authors refer to such distances as 'astro-kinematic' distances.

As anticipated above, the two samples are expected to trace a similar stellar population. For confirmation, we found that they have 265004 sources in common. Differences between the P18 and Z21 samples are presumably due to the cuts applied on photometry, which might exclude numerous late $B$-type stars in the UMS P18 sample, and astrometric quality cuts applied in the UMS Z21 sample. We tested the results presented in Sect. 3 using both UMS samples (and their corresponding different distance estimates to the stars) in order to verify the robustness of our findings.

\subsection{Open clusters}

We use the sample of open clusters with members and ages published by Cantat-Gaudin et al. (2020), which was based on Gaia DR2 data. All the member stars are brighter than $G=$ 18 and $98.4 \%$ of them have an EDR3 counterpart with the same source 'id' number, which we use to recover their EDR3 astrometry. In order to refine the mean astrometry of the clusters, including their mean parallax, we reject as members those stars whose EDR3 proper motion is discrepant from the cluster median by more than $3-\sigma$, and recompute the median EDR3 parallax.

Since the uncertainty on the median parallax of a cluster is much smaller than for individual stars, we estimate cluster distances by inverting their median EDR3 parallax after correcting for the known negative parallax zero point of $-17 \mu$ as. 
For this study we select the 687 open clusters younger than 100 Myr. Furthermore, we refine the selection by only considering the 353 open clusters with more than five members brighter than absolute magnitude $M_{G}=0$ (computed taking into account their distance and interstellar extinction). This additional cut has been applied to select intrinsically bright clusters, and compensate for observational biases, which otherwise would cause an over-sampling of clusters in the solar neighbourhood. In the following, we refer to this sample as the young and intrinsically bright open cluster data set. The complete open cluster sample can be downloaded from CDS.

\subsection{Classical Cepheids}

We compiled a list of classical Cepheids (DCEPs) taken from a variety of sources. In more detail, we adopted the list published by Gaia DR2 (Clementini et al. 2019) as revised by Ripepi et al. (2019), the compilation by Skowron et al. (2019), the recent new discoveries by the OGLE (Optical Gravitational Lensing Experiment Udalski et al. 2018; Soszyński et al. 2020) and ZTF (Zwicky Transient Facility, Chen et al. 2020) surveys. Merging the above catalogues, we obtained a sample of 2004 and 873 fundamental (DCEP_F) and first overtone (DCEP_1O) DCEPs, respectively. We note that the sample includes 238 mixed-mode pulsators that we assigned to DCEP_F or DCEP_1O on the basis of the dominant mode ( 85 and 153 DCEPs with dominant fundamental and 10 mode, respectively) ${ }^{1}$. The literature sample was cross-matched with EDR3 to obtain parallaxes and $G, G_{\mathrm{BP}}, G_{\mathrm{RP}}$ magnitudes (Riello et al. 2021). The correct average magnitude of a DCEP is normally calculated by integrating the light curve in intensity and then transformed back into magnitude. The Gaia magnitudes are calculated as arithmetic means and can differ from the "correct" ones by several hundredths of magnitudes (see e.g., Caputo et al. 1999). However, using DR2 results we estimate that for about $80 \%$ of the DCEPs the arithmetic mean of the Wesenheit magnitude defined in the text differs by less than 0.02 mag from the intensity-averaged one. In our case, the impact of such an uncertainty on the distance is generally negligible with respect to the uncertainty introduced by the parallaxes.

To derive the distances for our DCEP sample, we first calculated the individual Wesenheit magnitudes, which are particularly useful as they are reddening-free by definition (Madore 1982). The formulation for the apparent Wesenheit magnitude in the Gaia bands is $w=G-1.90 *\left(G_{\mathrm{BP}}-G_{\mathrm{RP}}\right)$ (Ripepi et al. 2019). Assuming the periods and modes from the literature, the individual DCEP distances (in parsecs) can be calculated directly from the distance modulus definition, $w-W=-5+5 * \log d(\mathrm{pc})$, where the absolute Wesenheit magnitude $\mathrm{W}$ is estimated through the period-Wessenheit (PW) relation. PW relations for both DCEP_F and DCEP_1O were published by Ripepi et al. (2019) based on Gaia DR2 parallaxes. However, the advent of the more precise EDR3 parallaxes led us to recalculate these relations, as described in Appendix A. Finally, the DCEP individual distances for the enlarged sample of 2004 DCEP_F and 873 DCEP_1O DCEPs were then calculated directly from the definition of distance modulus.

In the context of this paper, it is important to have a measure of the DCEPs age. These variables obey period-age (PA) and period-age-colour (PAC) relations (see e.g., Bono et al. 2005). In this work we estimated the ages using the updated PA rela-

\footnotetext{
1 The details of the construction of the sample will be given elsewhere (Ripepi et al. in prep.).
}

tion by De Somma et al. $(2020)^{2}$. In particular, we adopted the PA relations for models without overshooting, as this allows us to use both DCEP_F and DCEP_1O variables (see De Somma et al. 2020, for details). Using the PA relations, we select 1923 Cepheids with ages younger than $10^{8}$ years. These DCEPs have ages consistent with the young and intrinsically bright open clusters defined in Sect. 2.2.

\section{Results}

Figure 1 gives an overview of the UMS P18 data set. The spatial distribution of the stars in the $X-Y$ plane of the Galactic disc is presented in Fig. 1a, where the Sun is located at $(X, Y)=(0,0) \mathrm{kpc}$. The lack of stars near the position of the Sun $(\geqq 0.2 \mathrm{kpc})$ is due to our selection criteria, as mentioned in the previous section. The spatial distribution exhibits some dense clumps, which correspond to well-known OB associations, namely Cygnus, Carina, Cassiopeia, and Vela, amongst others. Also to be noted are radial features with respect to the Sun's position that become increasingly evident at heliocentric distances greater than about $2.5 \mathrm{kpc}$. These are artificial features that are not due to distance uncertainties, but are rather 'shadow cones' produced from foreground extinction in concert with the magnitude limit of this sample. For example, a prominent shadow cone is evident starting just beyond a dense clump of young stars at about $(X, Y)=(0,2) \mathrm{kpc}$ (Cygnus). In any case, any features showing a clear (radial or otherwise) symmetry with respect to the Sun's position should be treated with caution.

To make the spiral structure more evident, we map the stellar overdensity $\Delta_{\Sigma}$, defined as

$\Delta_{\Sigma}(X, Y)=\frac{\Sigma(X, Y)-\langle\Sigma(X, Y)\rangle}{\langle\Sigma(X, Y)\rangle}=\frac{\Sigma(X, Y)}{\langle\Sigma(X, Y)\rangle}-1$,

where $\Sigma(X, Y)$ is the local density at the position $(X, Y)$ in the Galactic plane, and $\langle\Sigma(X, Y)\rangle$ is the mean density. Both $\Sigma(X, Y)$ and $\langle\Sigma(X, Y)\rangle$ are calculated via a bivariate kernel density estimator, but using two different bandwidths, namely $0.3 \mathrm{kpc}$ and $2 \mathrm{kpc}$, respectively, for the local and mean density. Details on the calculation of $\Sigma(X, Y)$ and $\langle\Sigma(X, Y)\rangle$ are given in the Appendix B. The overdensity measured from the UMS P18 data set is shown in Fig. 1b. The corresponding plot using the UMS Z21 sample can be found in Fig. B.1. Both maps are very similar, though the UMS Z21 sample shows the inner Scutum arm more clearly, while the UMS P18 sample seems to have a better sampling of the Perseus arm.

To test the robustness of the obtained map, we also apply an alternative method using a wavelet transform to map overdense regions (details are given in Appendix B.2.). Figure 1c shows the obtained map, which is consistent with Fig. 1 b.

The global picture that emerges from Fig. 1 is that the distribution of the UMS stars is far from axisymmetric. The stars preferentially reside along arch-like features, which are hardly visible in the raw spatial distribution (Fig. 1a), but become quite evident when the over- and under-dense regions in the Galactic disc are mapped (Figs. 1b,c). These features in the spatial distribution of stars can be interpreted as coinciding with spiralarm segments in the vicinity of the Sun. Specifically, we discern three inclined red stripes in Figs. 1b,c, corresponding (from left to right) to the Perseus arm, the local arm nearest the Sun,

\footnotetext{
2 We preferred the PA over the PAC, as to use colours we would need to know the individual reddening values, which are rather uncertain for disc objects such as DCEPs.
} 

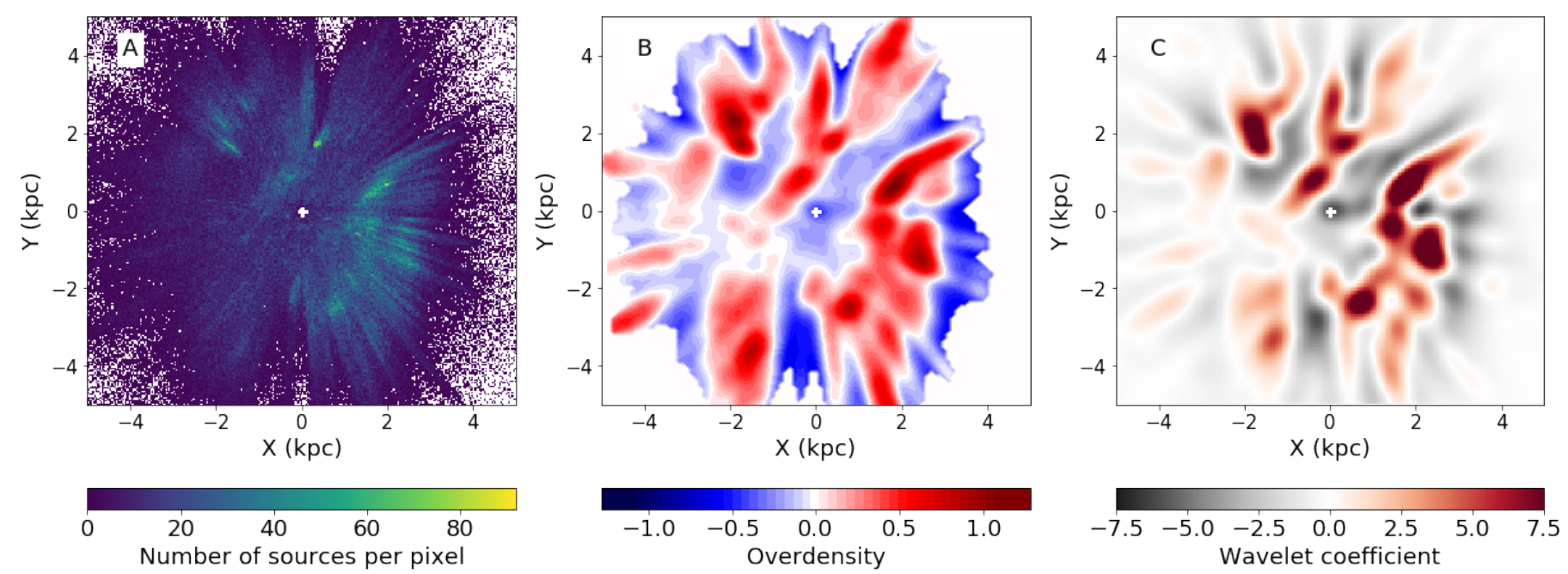

Fig. 1. Panel A: face-on view of the UMS P18 data set in the Galactic disc. The position of the Sun is shown by the white cross in $(X, Y)=(0,0)$. The Galactic centre is to the right, at $(X, Y)=\left(R_{\odot}, 0\right)$, and the Galaxy is rotating clockwise. Panel $B$ : same as panel A, but showing the measured overdensity using the UMS P18 data set, based on a local density scale length $0.3 \mathrm{kpc}$. Only points with $\Sigma(x, y)>0.003$ are plotted in order to remove regions where the statistics is too low. The corresponding plot for the UMS Z21 sample can be found in Fig. B.1. Panel C: same as panel A, but showing the wavelet transformation at the scale 3 (size $0.4 \mathrm{kpc}$ ). A different version of panels B and $\mathrm{C}$ using a larger scale length can be found in Fig. B.2. The maps shown in panels B and C are publicly available at https://github.com/epoggio/Spiral_arms_EDR3.git.

and an inner band to the Sagittarius-Carina and Scutum arms, respectively.

In Fig. 2, we dissect Fig. 1b into slices along the Galactocentric radius $R$, spaced by $10 \mathrm{deg}$ in Galactic azimuth. The assumed distance to the Galactic centre is $R_{\odot}=8.122 \mathrm{kpc}$ (Gravity Collaboration 2018). Each slice illustrates the measured overdensity features in the form of peaks or bumps as a function of $R$. We also compare the results obtained with the UMS P18 sample to the overdensities derived from the UMS Z21 data set. Notwithstanding the different selection criteria used to define the two UMS samples, and the different methods to calculate distances, Fig. 2 shows that most of the overdensity peaks are located at similar radii.

For a given slice, each peak in the overdensity can be typically identified with a spiral arm of the Milky Way. By comparing the $X Y$-map configuration (Fig. 2, top left) to the five slices, we can map the loci of the spiral arms throughout the Galactic plane.

The most external spiral-arm segment of the overdensity map, identified as the Perseus arm, is located at $R \sim 10 \mathrm{kpc}$ at $\phi \sim 20 \mathrm{deg}$, and extends in a clumpy but coherent way until $R \sim 12 \mathrm{kpc}, \phi \sim-10 \mathrm{deg}$. Much of this clumpiness is an artifact of foreground extinction, as evidenced by their radial orientation with respect to the Sun. At smaller Galactocentric radii, we find an overdensity arch-like feature that passes near the Sun, and extends from $R \sim 8 \mathrm{kpc}$ at $\phi \sim 20 \mathrm{deg}$ to $R \sim 10 \mathrm{kpc}$ at $\phi \sim-20$ deg. We identify this feature with the Local Arm. We also note that, at $\phi \sim 20 \mathrm{deg}$, the Local Arm exhibits a bifurcation, which might be an artifact caused by foreground extinction, as discussed above. In the innermost regions, we find the Sagittarius arm, extending from $R \sim 6 \mathrm{kpc}$ at $\phi \sim 10 \mathrm{deg}$ to $R \sim 8 \mathrm{kpc}$ at $\phi \sim-20 \mathrm{deg}$. It is not clear whether the overdensity found at $R \sim 5-5.5 \mathrm{kpc}$ and $\phi \sim 20 \mathrm{deg}$ corresponds to the Sagittarius or Scutum arm, which is the innermost spiral arm explored in this work. Scutum extends from $R \sim 5.5 \mathrm{kpc}$ at $\phi \sim 0$ deg to $R \sim 8 \mathrm{kpc}$ at $\phi \sim-30 \mathrm{deg}$.

In Fig. 2, uncertainties are calculated as described in Appendix B.3. As we can see, the statistical significance of the detected over- and under-densities at a given point strongly depends on the position in the Galactic plane. However, in this work, rather than focusing on the $\mathrm{S} / \mathrm{N}$ for each single point, we are more interested in understanding whether the observed global structures (i.e., the arch-like features mentioned above) are statistically significant. To this end, we construct a grid in the $(X, Y)$ plane, where points are spaced by $200 \mathrm{pc}$ along each coordinate. We then select the points of the grid lying along three spiral features identified in the overdensity map, that is (from left to right) the Perseus arm, the Local Arm, and the final one, which includes both the Sagittarius-Carina and Scutum arms (considered together here because they are difficult to clearly separate). We then construct wide bins of $10 \mathrm{deg}$ in Galactic azimuth $\phi$ for each spiral feature over its entire extent. Afterwards, for each feature, we check that the overdensity signal contained in all Galactic azimuth bins is significant, that is, it contains points with a $\mathrm{S} / \mathrm{N}$ greater than three. As can be noted, the features are clumpy, implying that the detected signal exhibits small-scale variations as a function of azimuth. Nevertheless, based on the procedure described above, we confirm that the global signal is statistically significant for all three features.

We then perform an additional test by calculating the statistical significance of the observed features using the wavelet transformation (Fig. 1c). To this end, we resort to the Multiresolution Analysis software (see Appendix B.2) and use it to compare the wavelet coefficients obtained from the data at each scale to the analytical distribution of coefficients that we would have obtained if our image had been flat (i.e., no signal) and affected by Poisson noise. We find that the identified spiral features in the UMS are more significant than $3 \sigma$, which is consistent with the results presented above.

The spiral structure detected with two UMS samples can then be compared to the distribution of other spiral-arm tracers. Figure 3 shows the spatial distribution of the young $(<100 \mathrm{Myr})$ and bright open clusters compared to the UMS overdensity map. Given the young age of the selected OCs, we expect them to be good tracers of their birthplaces. As we can see from Fig. 3, the distribution of the OCs is consistent with the overdense features found in the UMS sample, confirming that our UMS sample is 

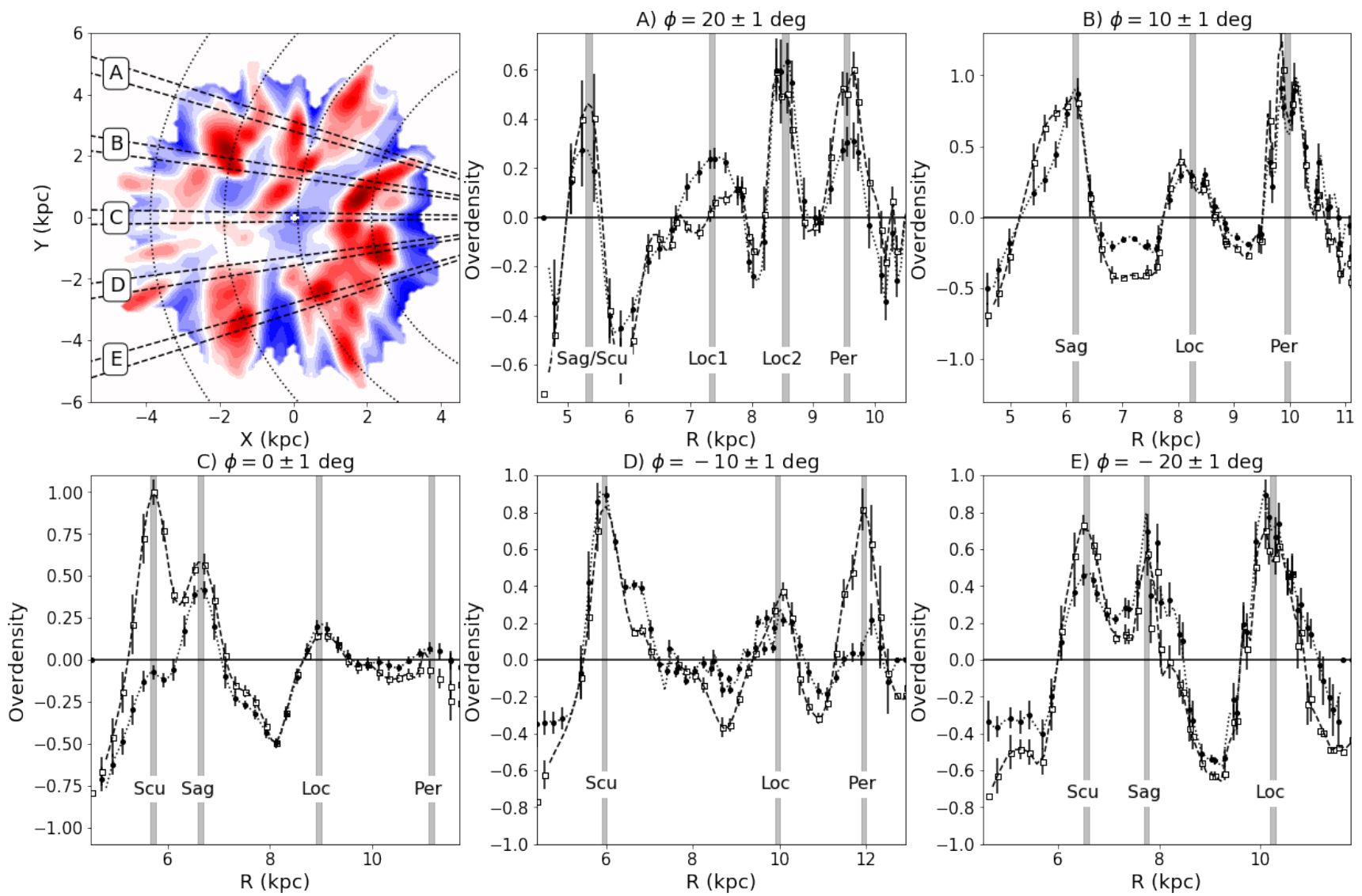

Fig. 2. Top left panel: same as Fig. 1b, but with some geometric references superimposed. Dotted lines from left to right show the points with constant Galactocentric radius $R=12,10,8,6 \mathrm{kpc}$, respectively. Dashed lines show the five selected slices (A,B,C,D,E), which are separated by $10 \mathrm{deg}$ in Galactic azimuth $\phi$ and by $2 \mathrm{deg}$ in width. Panels $A$ to E: profile of the measured overdensity as a function of $R$ for the UMS P18 sample (black dots) and the Z21 sample (white squares). Error bars show bootstrap uncertainties calculated as explained in Appendix B.3. For computational reasons, black dots/white squares and relative error bars were calculated for each slice and then connected using splines, as shown by the dotted (dashed) lines, to give a visual impression of how the overdensity varies at different radii. Each peak of the measured overdensity has been identified with a spiral arm in the Milky Way, as indicated by the vertical grey lines and the corresponding labels. The identification of each peak is not due to an assumed specific model, but is simply based on the geometric appearance of the top left panel (see text).

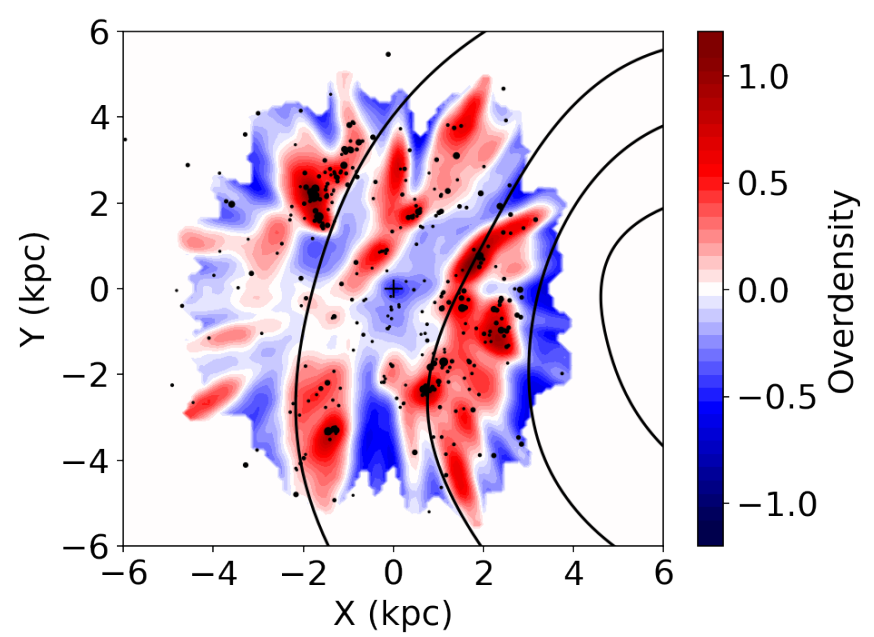

Fig. 3. Same as Fig. 1b, but compared to the distribution of the young and instrinsically bright open clusters sample (see Sect. 2.2) shown by the black dots. The size of the dots is proportional to the number of cluster members brighter than absolute magnitude $M_{G}>0$ (see text). Solid lines show the spiral arm model of Taylor \& Cordes (1993), which is based on HII regions. indeed young, and therefore tracing where star formation is more active.

Figure 4 (left panel) shows a comparison with the spatial distribution of the young $(<100 \mathrm{Myr})$ classical Cepheids. In contrast to the open clusters, within $5 \mathrm{kpc}$ of the Sun there is not an obvious correspondence between the UMS overdensity map and the distribution of Cepheids. This may be due in part to our sample of Cepheids being on average older than the open clusters, and therefore more dispersed and not as constrained to the spiral arms. Indeed, while both samples were selected to have ages of less than $100 \mathrm{Myr}$, the open cluster sample has a mean age of $45 \mathrm{Myr}$, while the mean age of the Cepheids is $66 \mathrm{Myr}$. Skowron et al. (2019) also show how the Cepheids diffuse with age. Also, while more numerous than the clusters, their individual distances (and ages) are less certain. It is for these reasons that we believe the Local Arm is not clearly evident in the Cepheid sample.

The advantage of the Cepheids is that they sample a much larger volume than the UMS stars and the open clusters, and we note that the UMS spiral arm segments might continue on a larger scale, as traced by the young Cepheids. In an effort to confirm this hypothesis, we produced an overdensity map of the Cepheids using the wavelet transform technique (Right panel of Fig. 4). The small sample size limits the resolution that can be 

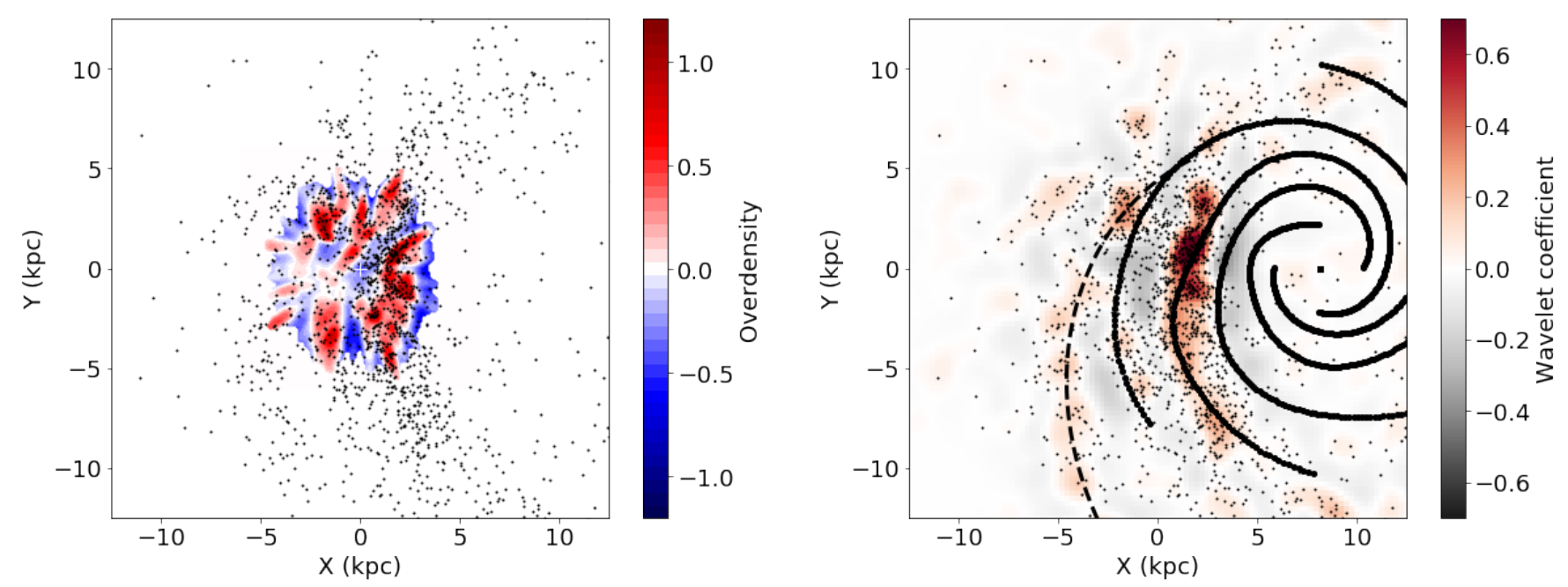

Fig. 4. Left panel: same as Fig. 1b, but on a larger scale, and compared to the distribution of the Cepheids sample (black dots). Right panel: wavelet transformation of the Cepheids sample, with the positions of the single Cepheids (black dots), the L06 model for the Perseus arm (dashed curve), and the spiral arm model of Taylor \& Cordes (1993) based on HII regions (solid lines) overplotted.

achieved (i.e., $1 \mathrm{kpc}$ ), meaning that the wavelet transform map cannot be built at the same scale as the UMS stars (i.e., $0.4 \mathrm{kpc}$ ), making the comparison between the two samples quite difficult. As in the left panel, the Local Arm is not evident near the Sun, though perhaps shows a weak feature in the third quadrant. Nevertheless, the Sagittarius-Carina (Sag-Car) arm is clearly evident (right panel). If we assume the Perseus arm model from Levine et al. (2006), we can see that it is traced out to a much larger distance from the Sun compared to the UMS sample. However, by estimating the statistical significance of the wavelet transform (in the same way as previously done for the UMS stars), we find that the signal of the Cepheids along the Perseus arm is only more significant than $3 \sigma$ in a few places, indicating a weaker signal compared to the one detected in the UMS sample, but nevertheless consistently tracing the proposed geometry of Levine et al. (2006) for this arm (see discussion below). The maps presented in this section are publicly available ${ }^{3}$.

\section{Discussion}

It is worth comparing our overdensity maps, which are based on the distribution of young UMS stars together with that of the open clusters and classical Cepheids, to previous models of the location of the spiral arms and other data sets available in the literature. Comparing our UMS overdensity map to the OB associations in the I and II quadrants first compiled by Morgan et al. (1953, see their Fig. 1) using updated distances from Benjamin (2021, priv. comm.), we find that there is excellent agreement (figure not shown here).

Figure 3 shows our UMS overdensity map with the spiral arm model based on positions of HII regions overlayed (Georgelin \& Georgelin 1976; Taylor \& Cordes 1993, hereafter TC93). The same model is also overlayed on the overdensity map of the Cepheids in Fig. 4, together with another model of the Perseus arm from Levine et al. (2006, hereafter L06), shown as a dashed line (arm n.2 in their paper). As mentioned in Sect. 1, this model is based on $21 \mathrm{~cm}$ emission from atomic hydrogen (HI), and covers the outer portions of the Galactic disc, which extends well outside the regions covered by our overdensity maps.

\footnotetext{
3 https://github.com/epoggio/Spiral_arms_EDR3.git
}

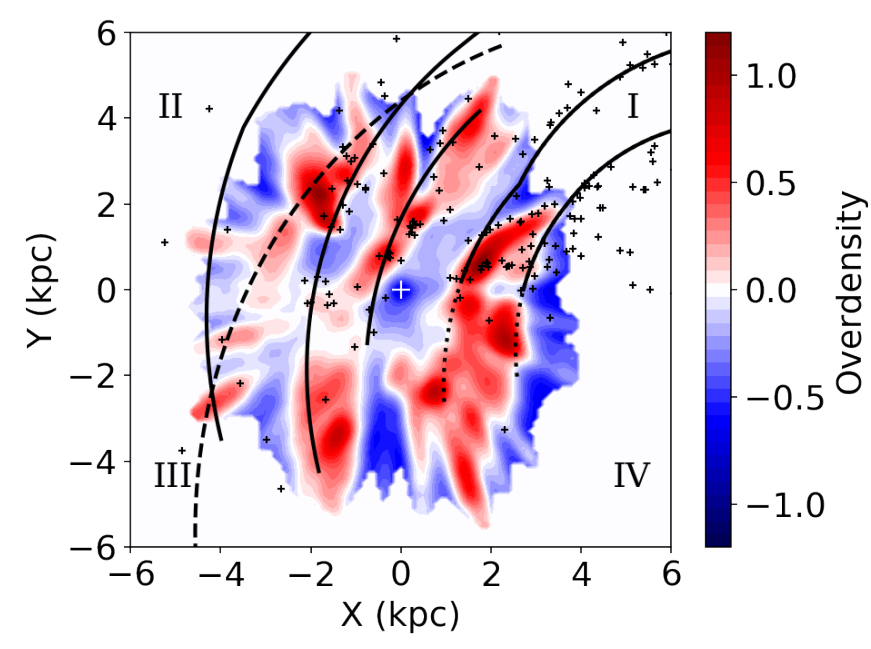

Fig. 5. Comparison between the measured overdensity map presented in this work (Fig. 1b), the distribution of the maser sources (black dots), the spiral arm model (solid lines, from left to right: Outer, Perseus, Local, Sagittarius-Carina, Scutum arm) from Reid et al. (2019), and the Perseus arm from Levine et al. (2006; dashed line). Roman numerals show the I, II, III, and IV Galactic quadrants.

Figure 5 shows a comparison between our overdensity map and the distribution of maser sources from Reid et al. (2019). We see that in the I and II Galactic quadrants $(Y>0)$ there is reasonably good correspondence between the UMS overdensity and the maser positions (black crosses). Unfortunately, because of the current lack of maser data, a comparison in the III and IV quandrants is not possible. Figure 5 also shows the position of the spiral arms according to the model proposed by Reid et al. (2019) (solid black curves, hereafter referred to as the R19 model), limited to the spatial range specified in their Table 2. For the two innermost arms (Sagittarius-Carina and Scutum), we show an extrapolation of their model (dotted lines), to explore the connection with our overdensity map. For a more extensive extrapolation of the Reid et al. (2019) model, see their Figs. 1 and 2.

As can be immediately seen, the UMS overdensity map shows a series of overdense regions that approximately follow the orientation of the Perseus arm as proposed by L06, with 
its relatively steep pitch angle, rather than that of R19 or that of TC93 (Fig. 3) based on HII regions. The distribution of the Cepheids, which extend significantly beyond the area of the disc covered by our overdensity map, also shows evidence of an outer structure consistent in orientation with that of the Perseus arm proposed by L06 (Fig. 4).

Another important difference between the R19 and TC93 models and the UMS overdensity in the III quadrant is the Local Arm. In our overdensity map, this arm seems to continue into the III quadrant, well beyond the limited extent that is usually proposed for this arm. Vázquez et al. (2008) and Xu et al. (2021) also both suggest that the Local arm extends further than previously thought, though they propose significantly different geometries: Xu et al. (2021) proposes that the Local Arm turns inward toward the Sag-Car arm, rather than into the III quadrant, while for Vázquez et al. (2008) it continues in the direction of galactic longitude $240^{\circ}$ until it connects to the Perseus arm. For the R19 and TC93 model, the main overdensity of UMS stars in the III quadrant, centred on $(X, Y)=(-1.75,-3) \mathrm{kpc}$, would be assigned to the Perseus arm. Indeed, it has been remarked that toward the Galactic anticentre there seems to be a large gap in the Perseus arm (Vázquez et al. 2008; Bukowiecki et al. 2011; Cantat-Gaudin et al. 2018, 2019). In our overdensity map, this gap is more naturally explained as simply being the inter-arm region between the Perseus arm, as traced by L06, and the Local arm extending into the III quadrant.

Finally, the overdensities associated with the Sag-Car arm in the I and IV quadrants $(X>0)$ appear to be in good agreement with the TC93 model based on the HII regions, as well as the extrapolation of this arm in the R19 model. Toward the Galactic centre we see a gap in the distribution of the UMS stars, clearly seen in the surface density (Fig. 1a), which might be the interarm gap between the Sag-Car arm and the inner Scutum arm. This innermost arm is better seen in the overdensity map of the Z21 sample (see Fig. B.1). However, the distance to this arm is closer to the Sun than either the R19 or TC93 models suggest. Alternatively, we are seeing another arm segment, possibly a branch off of the Sag-Car arm.

\section{Conclusions}

In this contribution, we present a study of the spiral structure of the Galactic disc as traced by UMS stars, young open clusters, and classical Cepheids using the new parallaxes in Gaia EDR3. We mapped the overdensity of the UMS stars using two different methods and two different samples, all leading to similar results. Three arm segments are clearly discerned, corresponding to the Perseus arm, the Local Arm, and the Sag-Car arm, while a short segment of the Scutum arm towards the Galactic centre is less evident, but more easily seen in the Z21 sample. Our resulting overdensity map for the UMS stars suggests that the geometry of the spiral arms in the III galactic quadrant differs significantly from those proposed by many current models, such as R19 and TC93, which are to a large degree based on an extrapolation of the arms as traced from sources in the I and II quadrants.

From the distribution of the UMS sample, we find that the Perseus arm follows the geometry proposed by Levine et al. (2006) based on HI radio data, and the distribution of Cepheids in the III quadrant is not inconsistent with this hypothesis. In addition, we see that the Local Arm in the UMS stars extends into the III quadrant at least $4 \mathrm{kpc}$ past the position of the Sun, giving it a full length of at least $8 \mathrm{kpc}$. This is still not enough to consider it a major arm of the Milky Way, but certainly means that it is an important spiral feature and more than a minor spur.
Finally, the Scutum arm appears to be closer to the Sun than previously thought.

The morphology of the spiral arms in the Galaxy is intrinsically connected to the mechanism responsible for their origin, which is still uncertain (Toomre 1977; Athanassoula 1984; Binney \& Tremaine 2008; Shu 2016). Therefore, the large-scale mapping of the spiral arms potentially contains important clues as to their nature, such as whether or not they were triggered by the passage of a satellite (Purcell et al. 2011; Laporte et al. 2018; Bland-Hawthorn \& Tepper-García 2021) and/or other mechanisms that might be at play (e.g., D’Onghia et al. 2013).

In any case, to discern the dynamical nature of the spiral arms of the Milky Way, kinematic information will also be needed, and in particular the in-plane systematic motions. Using stellar kinematics, Baba et al. (2018) found evidence that the Perseus arm is in the disruption phase of a transient arm. Recently, Pantaleoni González et al. (2021) identified a kinematically distinct structure, dubbed the Cepheus spur, extending from the Local arm towards the Perseus arm. This might be the same branch or spur off the Local Arm first noted by Morgan et al. (1953), and later mapped by Humphreys (1970). Pantaleoni González et al. (2021) suggest that this feature might be related to the Radcliffe wave (Alves et al. 2020), a coherent gaseous structure in the solar neighbourhood, extending for $2.7 \mathrm{kpc}$ in length. In the future, we can look forward to having line-of-sight spectroscopic velocities in the upcoming Gaia DR3 release to compliment the Gaia EDR3 astrometry.

While our distances are based on Gaia EDR3 astrometry, our UMS source lists are largely based on photometry from 2MASS. In the future, we can expect a significant increase in the volume of the disc sampled by Gaia thanks to the astrophysical parameters that are anticipated to be released in Gaia DR3 based on Gaia spectrophotometry and astrometry for stars as faint as $G=18$. The ability to select young populations at fainter magnitudes will push the limits of accurate Galactic cartography for young luminous stars to even greater distances.

Acknowledgements. We thank Robert Benjamin and Elena D'Onghia for fruitful discussions. This work has made use of data from the European Space Agency (ESA) mission Gaia (https://www. cosmos.esa.int/gaia), processed by the Gaia Data Processing and Analysis Consortium (DPAC, https: //www.cosmos.esa.int/web/gaia/dpac/consortium). Funding for the DPAC has been provided by national institutions, in particular the institutions participating in the Gaia Multilateral Agreement. This publication makes use of data products from the Two Micron All Sky Survey, which is a joint project of the University of Massachusetts and the Infrared Processing and Analysis Center/California Institute of Technology, funded by the National Aeronautics and Space Administration and the National Science Foundation. E. P. acknowledges support by the Centre national d'études spatiales (CNES). T. C. G., F. F., and M. R. G. acknowledge support by the Spanish Ministry of Science, Innovation and University (MICIU/FEDER, UE) through grants RTI2018095076-B-C21 and the Institute of Cosmos Sciences University of Barcelona (ICCUB, Unidad de Excelencia 'María de Maeztu') through grant CEX2019000918-M. R. B., Y. F., and A. L. acknowledge support by the BELgian federal Science Policy Office (BELSPO) through various PROgramme de Développement d'Expériences scientifiques (PRODEX) grants. R. A. and M. F. acknowledge the support from the DLR (German space agency) via grant 50 QG 1403. P. R. acknowledges support by the Agence Nationale de la Recherche (ANR project SEGAL ANR-19-CE31-0017), funding from the project ANR18-CE31-0006 and from the European Research Council (ERC grant agreement No. 834148). L. C. acknowledges support by the ANID/FONDECYT Regular Project 1210992. R. D., G. C., V. R. and T. M. acknowledge the support of the Italian Space Agency (ASI) for their continuing support through contract 201824-HH.0 to the National Institute for Astrophysics (INAF).

\section{References}

Alves, J., Zucker, C., Goodman, A. A., et al. 2020, Nature, 578, 237 
Antoja, T., Ramos, P., Mateu, C., et al. 2020, A\&A, 635, L3

Athanassoula, E. 1984, Phys. Rep., 114, 319

Baba, J., Kawata, D., Matsunaga, N., Grand, R. J. J., \& Hunt, J. A. S. 2018, ApJ, 853, L23

Binney, J., \& Tremaine, S. 2008, Galactic Dynamics: Second Edition (Princeton Princeton University Press)

Bland-Hawthorn, J., \& Maloney, P. R. 2002, ASP Conf. Ser., 254, 267

Bland-Hawthorn, J., \& Tepper-García, T. 2021, MNRAS, 504, 3168

Bono, G., Marconi, M., Cassisi, S., et al. 2005, ApJ, 621, 966

Bukowiecki, Ł., Maciejewski, G., Konorski, P., \& Strobel, A. 2011, Acta Astron., 61, 231

Cantat-Gaudin, T., Jordi, C., Vallenari, A., et al. 2018, A\&A, 618, A93

Cantat-Gaudin, T., Krone-Martins, A., Sedaghat, N., et al. 2019, A\&A, 624, A126

Cantat-Gaudin, T., Anders, F., Castro-Ginard, A., et al. 2020, A\&A, 640, A1

Caputo, F., Marconi, M., \& Ripepi, V. 1999, ApJ, 525, 784

Chen, B. Q., Huang, Y., Hou, L. G., et al. 2019, MNRAS, 487, 1400

Chen, X., Wang, S., Deng, L., et al. 2020, ApJS, 249, 18

Christiansen, W. N., \& Hindman, J. V. 1952, Aust. J. Sci. Res. A Phys. Sci., 5, 437

Clementini, G., Ripepi, V., Molinaro, R., et al. 2019, A\&A, 622, A60

De Somma, G., Marconi, M., Cassisi, S., et al. 2020, MNRAS, 496, 5039

D’Onghia, E., Vogelsberger, M., \& Hernquist, L. 2013, ApJ, 766, 34

Eilers, A.-C., Hogg, D. W., Rix, H.-W., et al. 2020, ApJ, 900, 186

Evans, D. W., Riello, M., De Angeli, F., et al. 2018, A\&A, 616, A4

Feigelson, E. D., \& Babu, G. J. 2012, Modern Statistical Methods for Astronomy (Cambridge: Cambridge University Press)

Gaia Collaboration (Brown, A. G. A., et al.) 2016, A\&A, 595, A2

Gaia Collaboration (Brown, A. G. A., et al.) 2018a, A\&A, 616, A1

Gaia Collaboration (Katz, D., et al.) 2018b, A\&A, 616, A11

Gaia Collaboration (Brown, A. G. A., et al.) 2021, A\&A, 649, A1

Georgelin, Y. M., \& Georgelin, Y. P. 1976, A\&A, 49, 57

Gravity Collaboration (Abuter, R., et al.) 2018, A\&A, 615, L15

Humphreys, R. M. 1970, AJ, 75, 602

Kerr, F. J., Hindman, J. V., \& Carpenter, M. S. 1957, Nature, 180, 677

Khoperskov, S., Gerhard, O., Di Matteo, P., et al. 2020, A\&A, 634, L8

Kounkel, M., Covey, K., \& Stassun, K. G. 2020, AJ, 160, 279

Laporte, C. F. P., Johnston, K. V., Gómez, F. A., Garavito-Camargo, N., \& Besla, G. 2018, MNRAS, 481, 286

Levine, E. S., Blitz, L., \& Heiles, C. 2006, Science, 312, 1773

Lindegren, L., Bastian, U., Biermann, M., et al. 2021, A\&A, 649, A4

Madore, B. F. 1982, ApJ, 253, 575

Miyachi, Y., Sakai, N., Kawata, D., et al. 2019, ApJ, 882, 48
Morgan, W. W., Sharpless, S., \& Osterbrock, D. 1952, AJ, 57, 3 Morgan, W. W., Whitford, A. E., \& Code, A. D. 1953, ApJ, 118, 318 Oort, J. H., Kerr, F. J., \& Westerhout, G. 1958, MNRAS, 118, 379 Pantaleoni González, M., Maíz Apellániz, J., Barbá, R. H., \& Reed, B. C. 2021 , MNRAS, 504, 2968

Poggio, E., Drimmel, R., Smart, R. L., Spagna, A., \& Lattanzi, M. G. 2017, A\&A, 601, A115

Poggio, E., Drimmel, R., Lattanzi, M. G., et al. 2018, MNRAS, 481, L21

Purcell, C. W., Bullock, J. S., Tollerud, E. J., Rocha, M., \& Chakrabarti, S. 2011, Nature, 477, 301

Ramos, P., Antoja, T., \& Figueras, F. 2018, A\&A, 619, A72

Ramos, P., Antoja, T., Mateu, C., et al. 2021, A\&A, 646, A99

Reid, M. J., Menten, K. M., Brunthaler, A., et al. 2014, ApJ, 783, 130

Reid, M. J., Menten, K. M., Brunthaler, A., et al. 2019, ApJ, 885, 131

Riello, M., De Angeli, F., Evans, D. W., et al. 2021, A\&A, 649, A3

Riess, A. G., Casertano, S., Yuan, W., et al. 2018, ApJ, 861, 126

Ripepi, V., Molinaro, R., Musella, I., et al. 2019, A\&A, 625, A14 Russeil, D. 2003, A\&A, 397, 133

Rybizki, J., Green, G., \& Rix, H.W. 2021, ArXiv e-prints [arXiv:2101.11641]

Shen, J., \& Zheng, X.-W. 2020, Res. Astron. Astrophys., 20, 159

Shu, F. H. 2016, ARA\&A, 54, 667

Skowron, D. M., Skowron, J., Mróz, P., et al. 2019, Science, 365, 478

Skrutskie, M. F., Cutri, R. M., Stiening, R., et al. 2006, AJ, 131, 1163

Soszyński, I., Udalski, A., Szymański, M. K., et al. 2020, Acta Astron., 70, 101

Starck, J. L., \& Murtagh, F. 2002, in Astronomical image and dataanalysis, eds. J. L. Starck, \& F. Murtagh (Springer)

Starck, J.-L., Murtagh, F. D., \& Bijaoui, A. 1998, Image Processing and Data Analysis (New York: Cambridge University Press), 297

Taylor, J. H., \& Cordes, J. M. 1993, ApJ, 411, 674

Toomre, A. 1977, ARA\&A, 15, 437

Udalski, A., Soszyński, I., Pietrukowicz, P., et al. 2018, Acta Astron., 68, 315

Vallée, J. P. 2005, AJ, 130, 569

van de Hulst, H. C., Muller, C. A., \& Oort, J. H. 1954, Bull. Astron. Inst. Netherlands, 12, 117

Vázquez, R. A., May, J., Carraro, G., et al. 2008, ApJ, 672, 930

Veselova, A. V., \& Nikiforov, I. 2020, Res. Astron. Astrophys., 20, 209

Virtanen, P., Gommers, R., Oliphant, T. E., et al. 2020, Nat. Meth., 17, 261

Xu, Y., Reid, M., Dame, T., et al. 2016, Sci. Adv., 2, e1600878

Xu, Y., Hou, L.-G., \& Wu, Y.-W. 2018a, Res. Astron. Astrophys., 18, 146

Xu, Y., Bian, S. B., Reid, M. J., et al. 2018b, A\&A, 616, L15

Xu, Y., Hou, L. G., Bian, S. B., et al. 2021, A\&A, 645, L8

Zari, E., Rix, H. W., Frankel, N., et al. 2021, A\&A, 650, A112

Zinn, J. C. 2021, AJ, 161, 214 


\section{Appendix A: New calibration of Cepheid PW relation}

To recalibrate the PW relations for the classical Cepheids, we made an additional refined selection of the Cepheid sample, retaining only objects with the most secure subclassification in modes from the literature (Ripepi et al. in prep.) and ruwe parameter $<1.4^{4}$, remaining in the end with a sample of wellcharacterized 852 and 396 DCEP_F and DCEP_1O, respectively. Then, we corrected the zero point offset of the parallaxes following the indications by Lindegren et al. (2021). However, several parametrisations are available in the literature. Zinn (2021) found an offset of $15 \mu$ as for sources with $G<10.8$, which may lead to a systematic error of up to $10 \%$ in our Cepheids sample. By testing such an impact, we found that it does not affect our conclusions on the spatial distribution of the Cepheid sample. Finally, we fitted the PW relation to the data in the form $W=a+b\left(\log _{10} P-P_{0}\right)$, where $W$ is the absolute Wesenheit magnitude, $P$ the period of each DCEP, and $P_{0}$ is a pivoting period equal to $1.0 \mathrm{~d}$ and $0.3 \mathrm{~d}$ for DCEP_F and DCEP_1O, respectively. Different relations were searched for $F$ and 10 mode DCEPs. To estimate the parameters of the PW relations, we adopted the formalism by Riess et al. (2018), which allows us to use the parallaxes in a linear way, keeping the symmetry of their uncertainty and avoiding biases from the cuts in parallax values. According to the distance modulus definition, we can define a photometric parallax (in mas),

$\varpi_{\text {phot }}=10^{-0.2(w-W-10)}$,

where $W$ is the absolute Wesenheit magnitude found from the PW relation and $w$ is defined in Sect. 2.3. Indicating with $\varpi_{\mathrm{EDR} 3}$ the zero-point corrected parallax from EDR3, we minimise the following quantity:

$\chi^{2}=\sum \frac{\left(\varpi_{\mathrm{EDR} 3}-\varpi_{\mathrm{phot}}\right)^{2}}{\sigma^{2}}$

where $\sigma$ is calculated by summing in quadrature the error on EDR3 parallaxes, the uncertainty of 0.01 mas on the parallax correction (Lindegren et al. 2021), the intrinsic dispersion of the PW relations (taken from Ripepi et al. 2019), and the photometric uncertainty on the apparent $w$, which we assumed conservatively fixed and equal to $0.03 \mathrm{mag}$ (more details on the whole procedure can be found in Ripepi et al. in prep.). We note that we did not consider any dependence on metallicity, as this information is missing for the large majority of the DCEPs considered here. Finally, we arrive at the following PW relations:

$W_{F}=(-6.015 \pm 0.005)-(3.317 \pm 0.028)\left(\log _{10} P_{F}-1.0\right)$,

$W_{1 O}=(-4.170 \pm 0.005)-(3.624 \pm 0.017)\left(\log _{10} P_{1 O}-0.3\right)$,

where the errors on the parameters are the formal output of the minimisation routine ${ }^{5} . P_{F}$ and $P_{10}$ are the periods of the $F$ and 10 mode pulsators, respectively. The DCEP_F PW relation is very similar to that derived by Ripepi et al. (2019) based on DR2 parallaxes, but with much smaller errors due to both a larger number of objects and more precise parallaxes. On the contrary, the relation for DCEP_10 is discrepant with respect to that from DR2, but in the latter case the errors were very large due to the small statistics (the present DCEP_1O sample is almost three times that used by Ripepi et al. 2019).

\footnotetext{
4 Section 14.1.2 of "Gaia Data Release 2 Documentation release 1.2"; https://gea.esac.esa.int/archive/documentation/GDR2/

5 (Scipy.optimize.minimize python package Virtanen et al. 2020).
}

\section{Appendix B: Adopted techniques}

\section{B.1. Bivariate kernel density estimation}

For a given position $(X, Y)$ in the Galactic plane, we calculate the local density $\Sigma(X, Y)$ through a bivariate kernel density estimator (following Eq. (6.11) in Feigelson \& Babu 2012), starting from the $\left(x_{i}, y_{i}\right)$-coordinates of the $N$ stars in our UMS sample, where $i=1, \ldots, N$ :

$\Sigma(X, Y)=\frac{1}{N h^{2}} \sum_{i=1}^{N}\left[K\left(\frac{X-x_{i}}{h}\right) K\left(\frac{Y-y_{i}}{h}\right)\right]$,

where $K$ is the kernel function and $h$ is the kernel bandwidth. Given the symmetry of the problem under study, here we adopt the same kernel function and the same bandwidth for both $X$ - and $Y$ - coordinates. In this paper, we used the following Epanechnikov kernel function

$K\left(\frac{X-x_{i}}{h}\right)=\frac{3}{4}\left(1-\left(\frac{X-x_{i}}{h}\right)^{2}\right)$,

for $\left|\left(X-x_{i}\right) / h\right|<1$, and is zero outside (and similarly for the $Y$-coordinate). However, we also tested other kernel functional forms, such as the triangle and Gaussian kernel (see e.g., Feigelson \& Babu 2012) to check that the choice of the kernel does not significantly influence the obtained maps. The figures presented in the main text are based on a local density bandwidth $h=0.3 \mathrm{kpc}$. Here we also show the results obtained with a local density bandwidth $h=0.8 \mathrm{kpc}$ (Fig. B.2).

To estimate the mean density $\langle\Sigma(X, Y)\rangle$, we use the same approach adopted for the local density (Eq. (B.1)), but choose a larger bandwidth $h=2 \mathrm{kpc}$. Afterwards, the local and mean density are combined, following Eq. (1), to obtain the stellar overdensity at a given position $(X, Y)$.

Additionally, we tested the effects of replacing the abovedescribed mean density with the raw average of the local densities calculated within $2 \mathrm{kpc}$ from each point. The resulting maps are consistent with the ones obtained above.

\section{B.2. Wavelet transformation}

The 2D wavelet transformation (WT, Starck \& Murtagh 2002) allows us to decompose an image into a set of layers, each one preserving only the structures with a characteristic size comparable to the wavelength of that layer (or scale). In terms of Fourier transformations, each layer of the WT can be roughly understood as the result of convolving the input with a pass-band that filters out those features in the original image that do not have the correct frequency.

There are many types of WT but, for the purpose of this work, we used the stationary wavelet transformation, also known as à trous algorithm, in its redundant form and using the $B$-spline mother wavelet. We computed the WT using the Multiresolution Analysis software (Starck et al. 1998), which has already been applied successfully to other astrophysical problems (for more details on the WT transform see, e.g., Ramos et al. 2018; Antoja et al. 2020; Ramos et al. 2021). Equation (B.3) shows the mathematical expression of the decomposition

$I(x, y)=c_{p}(x, y)+\sum_{j=1}^{p} \omega_{j}(x, y)$,

where $I$ represents the original image, $c_{p}$ is an extremely smoothed version of it, and $\omega_{j}$ are the arrays of wavelet coefficients at each layer $j \in[0, p]$. 


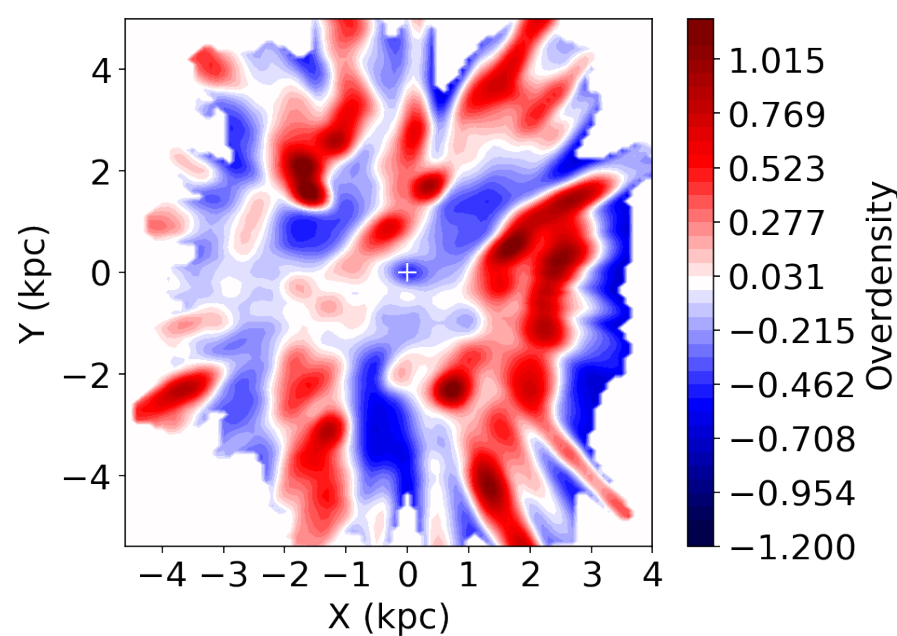

Fig. B.1. Same as Fig. 1b, but using the Z21 UMS sample.

The advantage of using this implementation of the WT in front of others is that the number of pixels are preserved and that each scale has zero mean. As a consequence of the latter, positive coefficients are directly related to overdensities and negatives ones to underdensities, which is why panels A and B of Fig. B.2 appear visually similar.

\section{B.3. Uncertainties}

As the overdensity $\Delta_{\Sigma}(X, Y)$ is a derived quantity (Eq. (1)), we calculate its uncertainty by first deriving the ones on the local
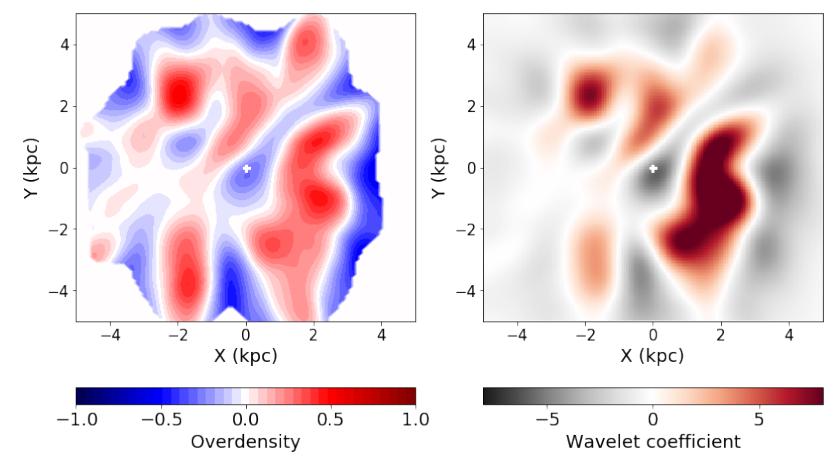

Fig. B.2. Same as Figs. 1b (left panel) and c (right panel), but using a larger scale length $h=0.8 \mathrm{kpc}$, which corresponds to scale $=4$ of the wavelet transform.

density $\Sigma(X, Y)$ and the mean density $\langle\Sigma(X, Y)\rangle$. To this end, for a given position $(X, Y)$, we generate 100 bootstrap resamples using the stars within 0.3 and $2 \mathrm{kpc}$, respectively, for the local and mean density. Using the resamples, we calculate the bootstrap standard deviation $\sigma_{\Sigma(X, Y)}$ and $\sigma_{\langle\Sigma(X, Y)\rangle}$. The uncertainty on the overdensity $\Delta_{\Sigma}(X, Y)$ is then derived via error propagation:

$$
\begin{aligned}
\sigma_{\Delta_{\Sigma}(X, Y)} & =\sqrt{\left(\frac{\partial \Delta_{\Sigma}(X, Y)}{\partial \Sigma(X, Y)} \sigma_{\Sigma(X, Y)}\right)^{2}+\left(\frac{\partial \Delta_{\Sigma}(X, Y)}{\partial\langle\Sigma(X, Y)\rangle} \sigma_{\langle\Sigma(X, Y)\rangle}\right)^{2}} \\
& =\sqrt{\left(\frac{\sigma_{\Sigma(X, Y)}}{\langle\Sigma(X, Y)\rangle}\right)^{2}+\left(\frac{\Sigma(X, Y)}{\langle\Sigma(X, Y)\rangle^{2}} \sigma_{\langle\Sigma(X, Y)\rangle}\right)^{2}}
\end{aligned}
$$

